\title{
Autologe Stammzelltransplantation Optimale Mobilisierungsstrategien für den individuellen Patienten
}

\begin{abstract}
Die autologe Stammzelltransplantation ist eine etablierte Therapie bei Patienten mit multiplem Myelom oder Lymphomen. Für einen optimalen Therapieerfolg ist eine ausreichende Stammzellmobilisierung essentiell. Ist die Mobilisierung mit dem Granulozyten-Kolonien stimulierenden Faktor (G-CSF) nach Induktionschemotherapie nicht erfolgreich, kann die Stammzellernte mit zusätzlicher PlerixaforGabe gesteigert werden. Bei einem von der Firma Sanofi gesponserten Workshop gingen Prof. Dr. Kai Hübel, Uniklinik Köln, und Dr. Patrick Wuchter, Universitätsklinikum Heidelberg, im Rahmen des COSTEM (Controversies in Stem Cell Transplantation and Cellular Therapies)-Kongresses auf aktuelle Fragen der Stammzellmobilisierung ein.
\end{abstract}

Laut European Society for Blood and Marrow Transplantation (EBMT) wurden im Jahr 2013 an 658 Zentren in 48 Ländern nahezu 40000 Transplantationen bei fast 35000 Patienten durchgeführt [1]. Bei 43\% der Patienten wurde allogen und bei $57 \%$ autolog transplantiert. Die Indikationen waren hauptsächlich Leukämien (32\%) und lymphoide Neoplasien (57\%). Ein geringer Teil der Transplantationen erfolgte aufgrund von soliden Tumoren (4\%) und nicht malignen Erkrankungen (6\%).

\section{Strategien zur Stammzellmobilisierung für die autologe Stammzelltransplantation}

Die autologe Stammzelltransplantation wird in der Regel bei Patienten mit multiplem Myelom oder Lymphom nach einer myeloablativen Chemotherapie eingesetzt. In einem Positionspapier stellten 28 Experten der EBMT die besten Herangehensweisen zur Mobilisierung von Stammzellen aus dem peripheren Blut und die Optimierung der Sammlung bei schlechten Mobilisierern dar [2]. Zurzeit werden 3 Strategien zur Stammzellmobilisierung angewendet: die alleinige G-CSF-Mobilisierung, die Chemotherapie-basierte Mobilisierung mithilfe der krankheitsspezifischen Chemotherapie und schließlich die separate Chemotherapie-Mobilisierung. Vorteile der alleinigen G-CSF-Mobilisierung sei die relativ geringe Toxizität mit Knochenschmerzen und Kopfschmerzen als häufigsten Nebenwirkungen, kommentierte Hübel. Die Apherese könne belastbar nach 4-5 Tagen geplant werden. Zudem können die Patienten ambulant behandelt werden und die Kosten seien vergleichsweise gering. Nachteile seien die geringere CD34+-Zellzahlen in der Sammlung verglichen mit der kombinierten Gabe von G-CSF und Chemotherapie sowie variable Versagerquoten. Die Effektivität der Sammlung kann gegebenenfalls nebenwirkungsarm und unkompliziert durch den zusätzlichen Einsatz des CXC-Motiv-Chemokinrezeptor 4 (CXCR4)-Antagonisten Plerixafor erhöht werden. Plerixafor bindet selektiv und reversibel an CXCR4 und verhindert damit die Fixierung der Zellen an das Knochenmarkstroma. Dadurch können die CD34+-Zellen in das periphere Blut ausgeschwemmt werden [3].
Mit dem Einsatz einer Chemotherapie werden höhere Stammzellzahlen im Vergleich zur alleinigen G-CSF-Mobilisierung erreicht, es werden weniger Apheresesitzungen benötigt und es kann die zusätzliche Antitumoraktivität von Cyclophosphamid genutzt werden. Nachteilig ist der weniger gut vorhersagbare Zeitpunkt des höchsten CD34+-Spiegels im peripheren Blut, der innerhalb eines Zeitrahmens von 10-18 Tagen auftritt, und die damit verminderte Auslastung der Aphereseeinrichtung. Zudem sind mehr Toxizitäten zu erwarten, die bis hin zur Schädigung des Knochenmarks führen können und damit möglicherweise eine spätere Mobilisierung verhindern. Die Versagerquote werde durch eine zusätzliche Chemotherapie nicht erhöht, aber die Patienten müssen für die Applikation der Chemotherapie stationär aufgenommen werden und es sind tägliche Bluttests zur Beobachtung der CD34+-Zellmobilisierung notwendig, bemerkte Hübel. Insgesamt entstehen damit höhere Kosten und eine grössere Belastung des Patienten als mit der alleinigen G-CSF-Mobilisierung.

\section{Zusammenhang zwischen Menge an CD34+-Zellen und Überlebensprognose}

Laut den Leitlinien der American Society for Blood and Marrow Transplantation (ASBMT) kann die Apherese durchgeführt werden, wenn mehr als 10-15 CD34+-Zellen/ $\mu \mathrm{l}$ im peripheren Blut gemessen werden [4]. Für eine Transplantation wird ein Minimum von 2,0 × $10^{6} \mathrm{CD} 34+-Z e l l e n /$ kg Körpergewicht (KG) benötigt [5]. Die Empfehlung für ein ausreichendes Engraftment liegt bei $5 \times 10^{6} \mathrm{CD} 34+-Z$ Zllen/kg KG. Werden weniger als 2,0 × $10^{6} \mathrm{CD} 34+-Z$ ellen $/ \mathrm{kg}$ KG gesammelt, hat die Mobilisierung laut Definition der ASBMT versagt [6].

Je höher die transplantierte Zellzahl, desto schneller das Thrombozyten-Engraftment, so das Ergebnis eines Reviews von Studien zur optimalen Zelldosis bei autologer und allogener Transplantation (Abb. 1) [7]. Daten einer retrospektiven Datenbank-Analyse von 350 Patienten mit Non-Hodgkin-Lymphom (NHL) oder Hodgkin-Lymphom (HD) zeigten zudem ein besseres Gesamtüberleben der Super-Mo-
S. Karger

Medical and Scientific Publishers

Basel · Freiburg · Paris · London

New York · Chennai · New Delhi

Bangkok · Beijing · Shanghai

Tokyo · Kuala Lumpur

Singapore $\cdot$ Sydney

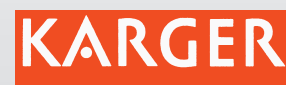

Beilage zu
Band 39, Heft 1-2, February 2016 


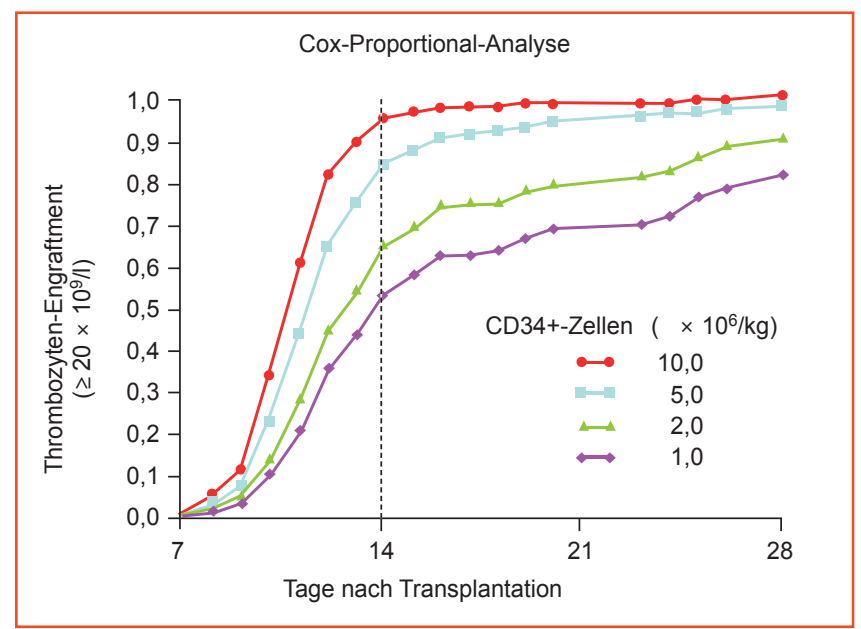

Abb. 1. Korrelation zwischen transplantierter CD34+-Zelldosis und Regenerationszeit der peripheren Blutzellen; modifiziert nach [6].

bilisierer, definiert als Patienten mit einer Stammzellernte $\geq 8 \times 10^{6}$ CD34+-Zellen/kg KG, im Vergleich mit Patienten, bei denen weniger als $8 \times 10^{6} \mathrm{CD} 34+-Z e l l e n / \mathrm{kg}$ KG mobilisiert wurden $(\mathrm{p}=0,005)$ [8]. In einer anderen retrospektiven Datenbankanalyse an 39 Patienten mit Multiplem Myelom (MM) oder Waldenström-Myelom wurde der Zusammenhang zwischen Überleben und der Anzahl mobilisierter CD34+-Zellen allerdings nicht bestätigt [9].

Aktuell bleibt der CD34+-Stammzellgehalt im Apharesat der wichtigste Parameter der Graftqualität, da es der einzige bekannte Prädiktor eines stabilen hämatopoetischen Engraftments nach Stammzelltransplantation ist. Im Workshop diskutierte Hübel mit den Teilnehmern, ob die Anzahl an Transplantationen, für die gesammelt wird, bei der ersten Mobilisierung entschieden werden soll. Die Handhabung erwies sich als durchaus verschieden: Während einige Zentren entsprechend des vorgefassten Plans die höchste mögliche Menge an Stammzellen transplantieren, heben andere Zentren überschüssige Stammzellen für weitere Transplantationen auf, auch wenn diese eigentlich nicht geplant sind.

Außerdem bleibe die Frage, ob die Chemomobilisierung als Mobilisierungsregime generell überdacht werden muss, da Patienten mit multiplem Myelom mit neuen Substanzen, wie Proteasominhibitoren oder immunmodulatorische Substanzen, und ihren Kombinationen mit deutlich besserem Ansprechen behandelt werden, ergänzte Hübel.

\section{Was ist die optimale Mobilisierungsstrategie? Einfluss, Herausforderungen und Lösungen}

Das Ziel sei es, so viele Stammzellen wie möglich in der bestmöglichen Qualität im ersten Mobilisierungsanlauf und in nur einer Leukapheresesitzung abzusammeln - und das möglichst nicht am Wochenende, formulierte Wuchter die heutigen Ansprüche an die Stammzellmobilisierung. Aktuelle Probleme sieht Wuchter allerdings bereits in der Charakterisierung des Patienten im Apheresealgorithmus: Beispielsweise würde bei der Definition der «schlechten Mobilisierer» die Frage nach dem individuellen Therapieziel nicht beachtet. Zudem sei die optimale Zeit der Stammzellmessung während der Mobilisierung und Kollektion nicht geklärt und es sei ebenfalls unklar, ob die Anzahl der CD34+-Zellen im peripheren Blut immer mit der Anzahl der gewonnenen Stammzellen korreliere.

Die Menge der zu sammelnden Stammzellen richtet sich nach der Anzahl der geplanten autologen Stammzelltransplantationen unter Berücksichtigung der geplanten Stammzelldosis, der Stammzellreserven und eines möglicherweise notwendigen Stammzellboosts. Die geringste akzeptable Anzahl an CD34+-Zellen beträgt laut EBMT 2,0 × 106/kg KG pro Transplantation [2]. Die therapeutische Strategie unterscheidet sich deutlich zwischen den weltweiten Transplantationszentren, bemerkte Wuchter und diskutierte die Vorgehensweisen und Erfahrungen mit den internationalen Workshop-Teilnehmern. Zumindest in Deutschland zeigte sich bei der Planung mehrerer Transplantationen das Problem der Zelldosis in Abhängigkeit vom Körpergewicht. Steigt das Körpergewicht des Patienten zwischen der ersten und zweiten Transplantation an, sind die eingelagerten Stammzellreserven möglicherweise per Definition nicht mehr ausreichend.

\section{Plerixafor erhöht die Stammzellernte bei schlechten Mobilisierern}

Erfahrungen aus Heidelberg aus den Jahren 2003 bis 2008 zeigten bei etwa $15 \%$ der 840 Patienten mit multiplem Myelom oder NHL eine schlechte Mobilisierung mit weniger als 20 CD34+-Zellen/ $\mu \mathrm{l}$ mit G-CSF-Stimulierung nach Induktionschemotherapie [10]. Als beeinflussende Faktoren für eine schlechtere Mobilisierung wurden in dieser Untersuchung die Anzahl vorangegangener Chemotherapie-Zyklen und eine vorherige Melphalan-Behandlung identifiziert. Positiv war hingegen die CD34+-Spitze im peripheren Blut mit der Menge geernteter CD34+-Zellen korreliert. Wuchter berichtete, dass im Universitätsklinikum Heidelberg bei schlechter Mobilisierung, d.h. bei einer CD34+-Zahl im peripheren Blut unterhalb von 10 Zellen/ $\mu$, präemptiv Plerixafor appliziert werde. In der «Grauzone» von 10-20 CD34+-Zellen/ $\mu$ l wird die Leukapherese evaluiert. Ist die Anzahl der geernteten CD34+-Zellen unterhalb eines Drittels des individuell geplanten Ernteziels, wird der Mobilisierungsversuch gestoppt oder eine Rescue-Mobilisierung mit Plerixafor durchgeführt (s. Abb. 2). Es sollten nicht mehr als 3 konsekutive Leukapheresesitzungen durchgeführt werden, um ein Transplant zu gewinnen, und insgesamt nicht mehr als maximal 4 Sitzungen veranschlagt werden. Wenn die Transplantation in 2 Tagen durchgeführt wird - mit maximal 2-3 Beuteln pro Tag - sollten zu hohe Transfusionsmengen an Dimethylsulfoxid (DMSO) pro Tag vermieden werden [11].

Die Rescue-Strategie mit Plerixafor hat sich in einer retrospektiven Untersuchung an 60 Patienten als effektiv erwiesen [12]. Es zeigte sich im Vergleich der Anzahl aller Apheresesitzungen pro Patient eine signifikant geringere Anzahl an Sitzungen an konsekutiven Tagen, um das Sammlungsziel zu erreichen, wenn Plerixafor eingesetzt wurde (s. Abb. 3). 
Plerixafor ist in Kombination mit G-CSF zur Verbesserung der Mobilisierung von hämatopoetischen Stammzellen im peripheren Blut bei erwachsenen Patienten mit Lymphom und multiplem Myelom, deren Zellen schlecht mobilisieren, zugelassen. Als Prädiktoren für eine schlechte Mobilisierung wurde das Alter (> 60 Jahre) und/oder eine vorherige myelosuppressive Chemotherapie und/oder eine extensive vorhergegangene Chemotherapie und/oder eine Spitzenzahl zirkulierender Stammzellen von weniger als $20 / \mu$ identifiziert. Die empfohlene Dosierung für Plerixafor beträgt $0,24 \mathrm{mg} / \mathrm{kg} \mathrm{KG}$ pro Tag. Es sollte 6-11 Stunden vor Beginn der Apherese subkutan nach 4 Tagen Vortherapie mit G-CSF appliziert werden. In der Diskussion mit den Workshop-Teilnehmern wurden auch kürzere Abstände zwischen Plerixafor-Applikation und Apherese vorgeschlagen, um die ambulante Versorgung innerhalb eines Tages zu gewährleisten.

\section{Neues Wissen zur Qualität der Stammzellmobilisierung}

Mit der Etablierung von Plerixafor und der damit gewonnenen Möglichkeit auch schlechte Mobilisierer einer autologen Stammzelltransplantation zuzuführen, verschiebt sich der Fokus von der Quantität mobilisierter CD34+ Stammzellen auch auf die Graftqualität. Die Graftzusammensetzung wird unter anderem von der Mobilisierungsstrategie beeinflusst: Im Vergleich von G-CSF versus G-CSF plus Plerixafor wurde unter der Plerixafor-Mobilisierung z.B. ein höherer Anteil an CD34-positiven/ CD38-negativen Zellen gesehen, wobei der $\mathrm{Zu}$ sammenhang dieser Zellkomponenten mit dem hämatopoetischen Engraftment unklar ist [13]. Ein größerer Anteil an T-Zellen und natürlichen Killerzellen (NK-Zellen) im Graft korreliert wahrscheinlich mit einem besseren Therapieerfolg.

In dem Zusammenhang müsse auch über den Einfluss von Cyclophosphamid, das häufig für die Mobilisierung verwendet werde, auf die Reduktion der Anzahl an T-, NK- und B-Zellen im Graft diskutiert werden, sagte Hübel. Vor allem bei intensiv vorbehandelten Patienten stelle sich die Frage nach der Effektivität einer Cyclophosphamid-basierten Mobilisierung. Unter der Mobilisierung mit Plerixafor wurde hingegen, unabhängig von der CD34+-Zellernte, eine erhöhte Mobilisierung von T- und NK-Zellen beobachtet [2]. Für den verbesserten Therapieerfolg sollten sowohl die hämatopoetische Regeneration als auch die Immunrekonstitution berücksichtigt werden. Beim B-Zell-Lymphom zeigte sich die Lymphozyten/Monozyten-Ratio im auto-Graft (A-LMR) als prognostischer Faktor für das Überleben nach autologer Stammzelltransplantation [14]. Zusammenfassend gelte für den derzeitigen Trend in der autologen Stammzelltransplan- tation, dass mit dem Überwinden der Hochdosis-Chemotherapie eine effektivere Immuntherapie möglich wird, konstatierte Hübel.

\section{Der Patient bleibt der wichtigste Parameter im Mobilisierungsprozess}

Bei der Planung einer autologen Stammzelltransplantation müsse vor allem auch der Standpunkt des Patienten beachtet werden, erinnerte Wuchter. So sei bei der Wahl des Mobilisierungsregimes auf Komorbiditäten und individuelle Besonderheiten des Patienten zu achten. Dazu gehöre auch die Frage der Anreise zur Klinik und der damit verbundenen Möglichkeiten einer ambulanten Versorgung. Um dem Patienten eine bessere Einsicht für anstehende Entscheidungen zu geben, könnten prädiktive Formeln in der Kommunikation mit dem Patienten die Unsicherheiten reduzieren, so die Erfahrung von Wuchter.

Für den Patienten scheint die ambulante Versorgung eine wichtige Rolle zu spielen, der heute Rechnung getragen werden müsse, so Wuchter. In Heidelberg würden etwa 90\% der 

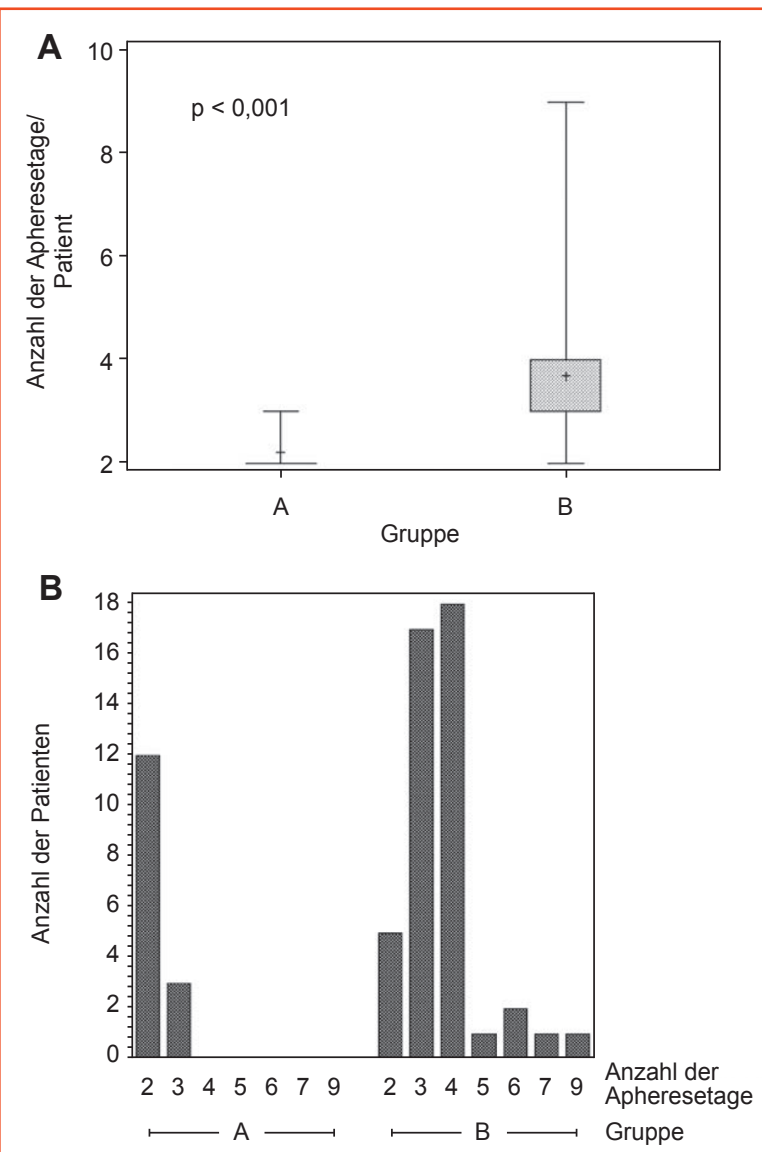

Abb. 3. Effektivität von Plerixafor als Rescue-Strategie; modifiziert nach [11].

Patienten für die Apherese nicht mehr stationär aufgenommen. Auch die Transplantation könne bei den meisten Patienten ambulant durchgeführt werden. In einer Befragung der Universität Heidelberg als Teil des Heidelberg Quality Management Surveys bei ambulant versorgten Patienten mit Hochdosis-Melphalan-Therapie gefolgt von einer autologen Stammzelltransplantation zeigten sich die Patienten sehr zufrieden mit der Behandlungsqualität, der medikamentösen Behandlung und der Betreuung. Alle Patienten waren gewillt, sich bei Bedarf einer zweiten Hochdosis-Chemotherapie mit autologer Stammzelltransplantation zu unterziehen. Patient sollten in jedem Fall über wiederholte Apheresesitzungen, die Möglichkeit eines Mobilisierungsfehlversuchs und die Remobilisierung aufgeklärt werden. Ebenso sollten die Patienten über die häufigsten Nebenwirkungen, wie Knochen- und Gelenkschmerzen, gastrointestinales Unbehagen und in seltenen Fällen Alopezie unter G-CSF bzw. Diarrhoe und Reaktionen an der Injektionsstelle unter Plerixafor, informiert sein. Auch bei der Leukapherese können, wenn auch selten, schwere Nebenwirkungen auftreten, die angesprochen werden sollten. Plerixafor habe wenige Nebenwirkungen und sei sehr effektiv, fasste Wuchter zusammen. Würde Plerixafor bei allen Patienten eingesetzt, wären die Algorithmen zur Stammzellmobilisierung weniger komplex und Plerixafor würde erheblich häufiger eingesetzt werden, sagte er.

\section{Zusammenfassung für die Praxis}

Die Definition des «schlechten Mobilisierers» ist komplex und hängt stark vom individuellen Ziel für die Stammzellernte ab. Der optimale Zeitpunkt für die Leukapherese ist essentiell für eine erfolgreiche Stammzellsammlung. Die Messung von CD34+-Zellen im peripheren Blut erlaubt es, Patienten mit erhöhtem Risiko für das Nicht-Erreichen des Sammlungsziels zu identifizieren. Plerixafor, ein selektiver und reversibler CXCR4-Rezeptoranatagonist, hemmt die Bindung der Stammzellen im Knochenmark und führt zu einer schnelleren und besseren Ausschwemmung der CD34+-Zellen ins periphere Blut. Bei Patienten, die nicht ausreichend mobilisieren, kann mit dem präemptiven Einsatz von Plerixafor die Stammzellsammlung verbessert und die Anzahl der notwendigen Apheresesitzungen verringert werden.

\section{Dr. Ine Schmale, Westerburg}

\section{Quelle}

Sanofi Industry Sponsored Workshop «How to find the optimal mobilization strategy? Impact, challenges and solutions», im Rahmen des COSTEMKongresses (Controversies in Stem Cell Transplantation and Cellular Therapies), 24. Oktober 2015, Berlin.

\section{Literatur}

1 Passweg JR et al.: Bone Marrow Transplant 2015;50:476-482.

2 Mohty M et al.: Bone Marrow Transplant 2014;49:865-872.

3 Fricker SP: Transfus Med Hemother 2013;40:237-245.

4 Duong HK et al.: Biol Blood Marrow Transplant 2014;20:1262-1273.

5 Giralt S et al.: Biol Blood Marrow Transplant 2014;20:295-308.

6 Pusic I et al.: Biol Blood Marrow Transplant 2008;14:1045-1056.

7 Siena S et al.: J Clin Oncol 2000;18:1360-1377.

8 Bolwell BJ et al.: Bone Marrow Transplant 2007;40:437-441.

9 Kakihana K et al.: Pathol Oncol Res 2010;16:583-587.

10 Wuchter P et al.: Biol Blood Marrow Transplant 2010;16:490-499.

11 Cheng J et al.: Transfusion 2015;55:275-283.

12 Hundemer M et al.: J Clin Apher 201;29:299-304.

13 Saraceni F et al.: Bone Marrow Transplant 2015;50:886-891.

14 Porrata LF et al.: Biol Blood Marrow Transplant 2014;20:1804-1812.

\section{Impressum}

Autologe Stammzelltransplantation

Optimale Mobilisierungsstrategien für den individuellen Patienten

Beilage zu Oncology Research and Treatment 39 | 1-2 | 16

Mit freundlicher Unterstützung durch

Sanofi-Aventis Deutschland $\mathrm{GmbH}$

Die Wiedergabe von Gebrauchsnamen, Handelsnamen, Warenbezeichnungen usw. in dieser Zeitschrift berechtigt auch ohne besondere Kennzeichnung nicht zur Annahme, dass solche Namen im Sinne der Warenzeichen- und Markenschutz-Gesetzgebung als frei zu betrachten wären und daher von jedermann benutzt werden dürfen. Für Angaben von Dosierungsanweisungen und Applikationsformen kann vom Verlag keine Haftung übernommen werden. Derartige Angaben müssen vom jeweiligen Anwender im Einzelfall anhand anderer Literaturstellen auf ihre Richtigkeit überprüft werden. Ohne schriftliche Genehmigung des Verlags dürfen diese Publikation oder Teile daraus nicht in andere Sprachen übersetzt oder in irgendeiner Form mit mechanischen oder elektronischen Mitteln (einschließlich Fotokopie, Tonaufnahme oder Mikrokopie) reproduziert oder auf einem Datenträger oder Computersystem gespeichert werden. Alle Rechte vorbehalten.

(c) 2016 by S. Karger Verlag für Medizin und Naturwissenschaften GmbH, Postfach, 79095 Freiburg, Deutschland.

Druck: Kraft Druck GmbH, 76275 Ettlingen, Deutschland. 